Théologiques

Théologiques

Dans la trace du bélier

La question de « l'animal » dans ce qui reste du judaiisme de Jacques Derrida

\title{
Orietta Ombrosi
}

Volume 22, numéro 1, 2014

URI : https://id.erudit.org/iderudit/1033098ar

DOI : https://doi.org/10.7202/1033098ar

Aller au sommaire du numéro

\section{Éditeur(s)}

Faculté de théologie et de sciences des religions, Université de Montréal

\section{ISSN}

1188-7109 (imprimé)

1492-1413 (numérique)

Découvrir la revue

Citer cet article

Ombrosi, O. (2014). Dans la trace du bélier : la question de «l'animal » dans ce qui reste du judaïsme de Jacques Derrida. Théologiques, 22(1), 111-142.

https://doi.org/10.7202/1033098ar

\section{Résumé de l'article}

Dans cet article, je cherche à envisager un certain judaïsme de Derrida ou ce qui reste du judaïsme dans sa pensée, en suivant les traces qu'un animal - le bélier - laisse dans les écrits du philosophe. En effet, les deux sujets, celui de l'animalité et celui de la judéité se croisent et se côtoient précisément autour de cette figure animale, qui n'est pas pourtant une figure ou une métaphore uniquement. Je choisis donc de suivre le bélier pour suivre l'adresse de Derrida qui appelle les philosophes à répondre philosophiquement de l'" animal ", c'est-à-dire à s'interroger de la " question animale ", d'une part ; d'autre part et en même temps, je le suis pour voir les moments essentiels les plus représentatifs dans le rituel juif, où cet animal se montre, et pour discerner ce qu'il révèle justement en relation au judaïsme tel que le philosophe Derrida l'a vécu ou conçu. L'article se divise donc en trois grandes parties qui correspondent aux trois scènes où le bélier, les béliers plutôt, fait/font son/leur apparition : la première scène est celle du bélier de la prière, se montrant et se cachant dans le tallith; ensuite, la scène sacrificielle par excellence (Gen 22) où le bélier du sacrifice est sacrifié par Abraham ; enfin, le bélier eschatologique, ainsi nommé parce qu'il est celui qui porte ou porterait l'autre, dans une dimension eschatologique à venir, selon une des dernières lectures derridiennes de Paul Celan, donnée dans le livre Béliers. 


\title{
Dans la trace du bélier
}

\section{La question de «l'animal» dans ce qui reste du judaïsme de Jacques Derrida}

\author{
Orietta Ombrosi* \\ Philosophie \\ Université Sapienza de Rome (Italie)
}

\begin{abstract}
Aux trois poules blanches, ensanglantées un jour quelconque, qui n'était même pas d'avant le Yom Kippur.
\end{abstract}

Autrefois, il n'y a pas longtemps, je cherchais et suivais un petit chat ${ }^{1}$, voire une chatte, dans les écrits de Jacques Derrida portant sur ce qui, par exemplification, est nommée la «question animale ${ }^{2}$ ». Je ne suis plus la seule depuis. Depuis, il y a eu aussi la parution d'autres écrits, d'autres livres signés par Derrida ou parus de manière posthume; il y a eu les gros volumes de ses derniers séminaires qui font trembler autant les bêtes que

* Orietta Ombrosi est maître de conférences, habilitée en philosophie morale au Département de philosophie de l'Université Sapienza de Rome. Ses recherches actuelles portent principalement sur J. Derrida et $\mathrm{S}$. Kofman. Elle a récemment publié en italien (2014) Il crepuscolo della ragione. Benjamin, Adorno, Horkheimer e Levinas di fronte alla Catastrofe, Giuntina (version originale française en 2007, traduction anglaise en 2012). Elle a aussi édité, avec A. Angerman, (2014, vol. 2) Critical Theory and Jewish Thougth pour la revue Bamidbar (Passagen).

1. Mon premier travail sur cette question a été écrit en 2008 (Ombrosi 2012).

2. Pour ce qui concerne la «question animale» dans la pensée de Derrida, il faut rappeler bien évidemment, le volume collectif L'Animal autobiographique (Mallet 1999 b) ; les textes consacrés à cette problématique dans certains livres: Fontenay (1998, 705-716 ; 2008, 15-40), Gontier (2011), Llored (2012); parmi les articles: Bensussan (2012), Grondin (2007), Michaud (2009) et le tout récent collectif Antonioli et Jabre (2014); pour l'Italie, voir Calarco (2012), Cimatti (2013) et le collectif Caffo et Ferraris (2014), entièrement dédié à la question de l'animalité chez Derrida. En anglais, au moins: Calarco (2008), Lawlor (2007), Berger (2011), Badmington et Wolfe (2007).

(C) Revue Théologiques 2014. Tout droit réservé. 
les souverains, ainsi que tous les loups que y sont convoqués et que le maître a voulu en quelque sorte domestiquer (Derrida, Lisse et al. 20082010). Ensuite, en suivant cette chatte qui était la sienne, je me suis entêtée à vouloir trouver une ânesse dans la "zootobiographie» de ce philosophe (Ombrosi 2010) - qui a eu le courage de mettre à l'ordre du jour pour les travaux généraux d'une philosophie à venir la question de la souffrance de ceux qu'il appelle "animot». Or, n'ayant pas trouvée cette ânesse - la docile et sage, la clairvoyante et humble monture de Balaam, qui voyait l'ange de YHWH et posait la question de la violence et de la souffrance infligées à elle, à ses semblables et dissemblables, selon le récit de $\mathrm{Nb} 22$, 22-35 - , je suis tombée sur une chimère, la grande. Sur la "chimère "invincible" " que Derrida a contemplée, admirée, suivie. Chimère guide, chimère tentation, chimère utopie. Femelle elle aussi, comme la chatte et l'ânesse. De genre féminin, d'où il en va aussi de la différence sexuelle et de la question du genre. Cette chimère, donc, m'a séduite. Elle m'a conduite jusqu'ici. Jusqu'au bélier, et dans sa trace. Elle m'a effrayé aussi.

Comme moi, avant moi, elle a foudroyé et effrayé Derrida, en le poussant à formuler le «discours le plus chimérique» qu'il ait jamais énoncé, celui concernant « les responsabilités, les obligations à l'égard du vivant en général ", selon ses mots, et touchant à «la compassion fondamentale qui, si on la prenait au sérieux devrait changer jusqu'au socle [...] la problématique philosophique de l'animal» (Derrida et Mallet 2006, 48). Or, cette chimère ou pensée chimérique qui, par sa bouche de feu, a enflammé une vénérable tradition et engendré une véritable "guerre» de religion, mieux, une guerre au nom de la pitié, "une guerre au sujet de la pitié », toujours selon les mots de Derrida (Derrida et Mallet 2006, 50), cette chimère donc semble être - en tout cas elle l'est pour moi qui l'ai aperçue et suivie — la plus grande bête que Derrida ait jamais connue ainsi que le plus grand défi que posent sa déconstruction et son héritage. "Khimaire m'intéresse, dit-il, car chimérique sera mon adresse» (Derrida et Mallet 2006, 66 - je souligne). Quelle adresse donc? Quel héritage chimérique? "Penser cette guerre dans laquelle nous sommes, écrit-il, ce n'est pas seulement un devoir, une responsabilité, une obligation, c'est aussi une nécessité, une contrainte à laquelle [...] nul ne saurait se soustraire. Désormais plus que jamais. Et si je dis "penser" cette guerre, c'est parce que je crois qu'il y va de ce que nous appelons "penser". L" "animal" nous regarde et nous sommes nus devant lui. Et penser commence peut-être là» (Derrida et Mallet 2006, 50 — je souligne). "Penser", pour le déconstructeur et défaiseur du logocentrisme d'abord, du phallogocentrisme ensuite et du carnophallogocen- 
trisme enfin, signifie donc: s'arrêter nu ${ }^{3}$, devant «l'animal»/« animot»; cela signifie aussi: s'arrêter pour le suivre et l'entendre, en suivant sa chimère, en allant derrière lui, derrière la question philosophique touchant à «l'animal » et à la chimérique responsabilité du penser — voire d'un logos décentré - vis-à-vis de celui-ci, ou de ceux qui très maladroitement sont nommés «animaux».

Or, je vais suivre Derrida encore, ici même, dans cette pensée du "devant "l'animal" ", qui est principalement un "derrière "l'animal" ", car il s'agit de suivre "l'animal", comme il nous l'a magistralement enseigné dans L'animal que donc je suis, en accueillant ainsi son héritage philosophique le plus chimérique et le plus fabuleux, certes, mais aussi le plus urgent et le plus vrai. "Sans doute» — peut-être, direz-vous. Toujours peut-être! Je vais donc suivre Derrida derrière cette chimère, sur son dos même, dos de chèvre - il faut le rappeler comme il le rappelle, car Chimère est «lion par-devant, serpent par-derrière et chèvre au milieu » (Derrida et Mallet 2006, 71) —, en planant au-dessus des mers abyssales et à travers les cieux orageux de la mythologie grecque où vit Chimère, pour descendre vers les paysages apparemment plus apaisants des déserts bibliques et y trouver le bélier, son cousin - cousin de la chèvre et non pas de la Chimère! D'un monstre mythologique, né de Thyphon et d'Echidna, dont nous parlent Homère et Plutarque, à un simple bélier, né d'un père bélier et d'une mère brebis, comme tant d'autres qui paissent et broutent dans les pages de la Bible. D'un «animot» à l'autre, d'une mythe à l'autre, mais aussi d'une tradition à l'autre, non tant pour souffler encore sur la finale bien connue de "Violence et métaphysique $e^{4}$, mais pour ressaisir ce virage animalier initial qui passe de la chimère au bélier, afin de poser à nouveau la/sa question: "Sommes-nous des Juifs? Sommes-nous des Grecs? Nous vivons dans la différence entre le Juif et le Grec. [...] Sommes-nous des Grecs? Sommes-nous des Juifs? Mais qui, nous? Sommes-nous (question non chronologique, question pré-logique) d'abord des Juifs ou d'abord des Grecs?» (Derrida 1967, 227). Mais qui, nous? Cette fois, c'est-à-dire ici, ce nous c'est lui, Jacques Derrida, mais aussi un peu le bélier... Derrida est-il d'abord Juif ou d'abord Grec? Car le bélier, lui, est certainement juif,

3. Ce discours sur la nudité serait à interroger davantage et impliquerait aussi un discours sur la pudeur; voir Derrida et Mallet (2006, 19.29.58-60.69-72), à comparer avec la nudité de Noé à propos de laquelle Derrida écrit dans Donner la mort (1999, 194-204). Voir aussi Cixous (2003, 79).

4. «Jewgreek is greekjew. Extremes meet» (Derrida 1967, 228, citant James Joyce et soulignant par l'italique). 
mais pas seulement...Voici donc que, par un coup de bélier précisément, nous sommes catapultés au milieu de la question que moi-même j'ai choisi de poser, voire de signer.

Dans la trace du bélier. J'y reviens, après ma petite histoire introductive, après avoir suivi les traces d'une chatte, d'une ânesse et avoir monté la chimère; j'y reviens, derrière cet "animal» — un mâle cette fois derrière sa trace parce que derrière lui se trace à mon sens ce qui reste d'un certain judaïsme de Derrida; mais j'y reviens et je le suivrai sans jamais abandonner l'adresse du philosophe qui nous appelle - nous, Juifs et Grecs, nous les philosophes en particulier - à répondre philosophiquement de l' «animal ». Je choisis donc de suivre le bélier et sa trace parce que celui-ci est l'un des animaux les plus représentatifs dans le rituel juif, et cela non seulement dans la scène sacrificielle, comme j'essayerai de le montrer, mais aussi parce que le bélier est celui qui porte ou porterait l'autre, dans une dimension eschatologique à venir, selon une des dernières lectures derridiennes de Paul Celan, donnée dans le livre intitulé Béliers (Derrida 2003a), justement, écrit un an avant sa mort. En effet, bien qu'apparemment méfiant, ce bélier ou plutôt ces béliers - au pluriel, il ne faut jamais oublier le pluriel en déconstruction! - se montre(nt) à différentes reprises/ étapes dans la topographie animalière derridienne et dans les textes les plus manifestement autobiographiques, se souvenant d'un certain judaïsme, ou le rappelant: à chaque fois, le(s) bélier(s) se montre(nt), bien que de manières singulières et en restant souvent en retrait, dans une relation toute à déchiffrer - avec la «judéité» ou, si l'on préfère, avec l' "être ou quasi-être-juif» de Derrida, "quasi-être-juif», écrit en un seul mot dans l'essai «Abraham, l'autre» (Derrida 2003b - texte écrit en 2000).

Mais auparavant, quelques pas préliminaires sur la trace. Restons dans la trace. Restons derrière. On sait très bien que la trace - ou mieux, le concept de trace - a été essentielle à Derrida pour la déconstruction du droit «logocentrisme». Il le rappelle clairement dans ce passage de L'animal que donc je suis:

si la déconstruction du «logocentrisme» a dû, tout nécessairement, se déployer à travers les années en déconstruction du "phallogocentrisme", puis du "carnophallogocentrisme », la substitution toute initiale du concept de trace ou de marque aux concepts de parole, de signe ou de signifiant était d'avance destinée, et délibérément, à passer la frontière d'un anthropocentrisme, la limite d'un langage confiné dans le discours et les mots humains. La marque, le gramme, la trace, la différance concernent différentiellement 
tous les vivants, tous les rapports du vivant au non-vivant. (Derrida et Mallet 2006, 144, je souligne).

Donc, la trace est avant tout non tant la signature de la déconstruction en acte, que la signature «de tous les vivants ", celle qui différentiellement signe leur différance et signe pour leur différance. Être dans la trace signifie en effet "être après, être auprès, être près de » (Derrida et Mallet 2006, 27), c'est-à-dire être avec — avec "l'animal »; mieux, cela signifie être proche de «l'animal» et, en quelque sorte, il faut encore en connaître l'ordre ou la façon, être son "prochain» (Derrida et Mallet 2006, 28). Alors, être après, être derrière, signifie donner le pas à «l'animal»/ «l'animot» et, plus simplement, le faire passer devant, devant tout le reste. Comme en ce moment même, dans l'acheminement des premiers pas de cet essai, le bélier passe devant. Devant moi aussi.

Restons alors encore derrière, sans le voir passer. Restons encore dans sa trace, mais sans le voir la tracer. Car c'est le propre de la trace, qu'elle soit celle du bélier, de l'homme ou encore de Dieu, de pouvoir toujours s'effacer, selon le propos central de Derrida dans sa critique à Jacques Lacan qui, au contraire, construit tout son argument quant à la «discontinuité radicale entre l'animal et l'humain" (Derrida et Mallet 2006, 184) autour de l'impossibilité pour le premier d'effacer ses traces. Si en effet, pour Lacan, il y a une opposition décisive entre le tracer et l'effacer des traces - cette seconde option étant une exclusivité de l'humain, puisqu'il est «sujet du signifiant» — en revanche, selon Derrida, c'est la structure de la trace elle-même qui suppose que «tracer revienne à effacer une trace (toujours présente-absente) autant qu'à l'imprimer» (Derrida et Mallet 2006, 185 $)^{5}$. Cela est démontré par de nombreuses pratiques animales, qu'elles soient rituelles ou accidentelles, par l'expérience de la feinte et de «la feinte de la feinte» que les "animaux» font également, par exemple, mais aussi par leur capacité/possibilité "d'effacer radicalement ses [leurs] traces, c'est-à-dire aussi bien de radicalement détruire, nier, mettre à mort, voire se mettre à mort» (Derrida et Mallet 2006, 186). L'effacement de traces n'implique ni un moi conscient ni un «sujet maître» qui puisse disposer du pouvoir de les effacer. Il appartient à la trace et donc aussi à celle de "l'animal», "de toujours s'effacer et de toujours pouvoir s'effacer» (Derrida et Mallet 2006, 186). En soulignant une sorte de «passivité » de

5. À propos de l'écriture comme trace et la possibilité de son effacement et de la perte de la trace du destinataire, voir aussi Derrida (1980). 
la trace dans l'italique apposé au pronom réflexif se, Derrida insiste beaucoup sur le non-ponvoir d'un quelconque sujet sur la trace, sur sa propre trace: «les traces $\left(s^{\prime}\right)$ effacent, comme tout, mais il appartient à la structure de la trace qu'il ne soit au pouvoir de personne de l'effacer ou surtout de “juger" de son effacement, encore moins d'un pouvoir constitutif assuré d'effacer, performativement, ce qui s'efface » (Derrida et Mallet 2006, 186). L'homme donc n'aurait pas plus de pouvoir que ledit "animal» quant à l'effacement des traces et cela détournerait déjà, au moins en peu, de l'anthropocentrique supériorité et sûreté de l'homme quant à l'"animot». Au moins, dis-je. Il ne s'agit que d'un moins, d'un petit rien: dans la question du "devant "l'animal" " en effet, et tout en restant derrière lui, dans sa/la trace, il ne faut pas uniquement se demander si on a le droit de lui refuser tel ou tel autre pouvoir ${ }^{6}$ - ici le pouvoir d'effacer ses traces mais il s'agit d'aller plus loin, en se demandant de quel droit l'homme s'attribue, lui, ce qu'il refuse à «l'animal». De quel droit, justement? Un droit sans justice, certainement.

Dans la trace du bélier, je reste donc et je le suis, même si celui-ci, comme les autres "animot", sans que je l'aie vu en détail et peut-être même à son insu, trace et efface ses traces. Il n'est donc pas facile de le suivre à la trace. Or, cette difficulté de le poursuivre vaut également pour le bélier en papier qui veille et fait ses apparitions entre les pages infinies des livres de Derrida. De même que le bélier de chair, de laine et de sabots, le bélier dans la trace duquel je suis, le bélier évoqué par Derrida, est craintif, peut-être endurci, donc difficile d'approche, à l'approche et il ne se montre pas très souvent; mieux, il ne se montre jamais spontanément bien qu'on le voie - je le vois - passer sur des scènes très décisives liées à la judéité de Derrida. Voici les scènes: une seule fois, mais tous les jours ou mieux tous les soirs, le bélier apparaît à peine sorti de l'armoire, sa cachette, un peu vivant bien que déjà mort, il se montre comme l'"autre peau », comme le tallith, comme le seul objet que Derrida ait jamais pos-

6. Il vaut la peine de citer ce passage: «Il ne s'agit pas seulement de demander si on a le droit de refuser tel ou tel pouvoir à l'animal (parole, raison, expérience de la mort, deuil, culture, institution, technique, vêtement, mensonge, feinte de la feinte, effacement de la trace, don, rire, pleur, respect, etc. - la liste est nécessairement indéfinie, et la plus puissante tradition philosophique dans laquelle nous vivons a refusé tout cela à "l'animal”). Il s'agit aussi de se demander si ce qui s'appelle l'homme a le droit d'attribuer en toute rigueur à l'homme, de s'attribuer, donc, ce qu'il refuse à l'animal, et s'il en a jamais le concept pur, rigoureux, indivisible, en tant que tel " (Derrida et Mallet 2006, 185-186, l'auteur souligne). 
sédé quant à la "chose “juive" " (Derrida 2003b, 15), il se montre donc dans l'intimité absolue de la prière et de la bénédiction — d'où mon choix de le surnommer "le bélier de la prière "; une autre fois, voire plusieurs fois, presque chaque fois, il se montre sur le mont Moriah, encore bien vivant et prêt à recevoir le couteau fatal d'Abraham à la place d'Isaac c'est «le bélier du sacrifice»; enfin, un autre bélier fait son apparition essentielle, recueillant aussi les destins des deux autres — comme les deux autres en fait -, celui qui se montre vivant sous la grande voûte incandescente du ciel celanien du poème Grosse, Glühende Wölbung (Grande Voûte Incandescente) du recueil Atemwende, où il est destiné à porter l'autre ou bien à être porté lorsque le monde n'est plus — je le nomme «le bélier de l'eschatologie».

\section{Première scène. «Le bélier de la prière »}

Dans «Un ver à soie » (écrit en 1995, publié en 1998) — dans ce texte unique, écrit dans la tentation du «défaire» à l'infini, par la «diminution» ou la «déconstruction", écrit aussi «dans la figure du textile» (Derrida 1998, 27) ou dans celle d'un "journal de voyage ", dans ce texte qui semble être tissé à la main et qui est aussi un "traité" non théologique de théologie, où il y est question de révélation, de voilement, de dévoilement, du voir, du toucher, mais aussi de foi et du savoir... - dans ce texte donc apparaît bel et bien le bélier, bien que transfiguré: il est dans le tallith, il est le tallith, le châle de la prière. Il faudrait relire ce texte mot après mot, page après page (Derrida 1998, en particulier 44-47, 62-69, 71-74, 79-85). Et déjà d'autres l'ont fait, bien qu'en partie seulement, les amies et commentatrices les plus proches de Derrida (Cixous 2001, 103-109; Malabou 2003; CalleGruber 1999; Mallet 1999a; Michaud 2010; Simma, dans Berger 20117). Diverses perspectives, différentes aussi de la mienne, mais en tous cas perspectives de femmes, peut-être fascinées par le tissu ou le tricot, ou par cette «confection tisserande et couturière » de Derrida (Calle-Gruber 1999, 77), ou mieux, voire ou pire, attirées par une sorte d'envie du tallith, comme une envie du phallus, étant donné que le tallith ainsi que la circoncision, «la blessure mortelle» (Derrida 1998, 62), comme le rappelle Derrida sans cesse et comme le rappelle à lui aussi son tallith, est «le propre de

7. J'aurais voulu entendre la lecture de ce texte par Sarah Kofman, mais elle n'était déjà plus là au moment de sa publication; elle nous aurait, certes, beaucoup appris, comme elle le fait d'ailleurs dans Lectures de Jacques Derrida (Kofman 1984). 
l'homme» (Derrida 1998, 46, 79). Mais, pour en rester à ma lecture et à ma chasse sans chasse du bélier, dans le tallith, s'agit-il encore de "l'animal » ? S'agit-il encore du bélier ? Derrida écrit:

Mon châle à moi. Le mien fut blanc d'abord, tout blanc, seulement blanc, vierge et sans ces bandes noires ou bleues qu'on imprime, me semble-t-il, sur presque tous les tallith du monde. Ce fut en tous cas le seul tallith blanc de la famille. Il me fut donné par le père de ma mère, Moïse. Comme en signe d'élection, mais pourquoi ? Pourquoi moi? Je dis qu'il fut blanc car avec le temps il devint un peu jaune. Je ne sais pas pourquoi, après que j'eus quitté la maison d'El Biar [...]. Je ne le porte presque jamais (est-ce le bon mot, porte? porte-t-on cette chose? en a-t-elle besoin? n'emporte-t-elle pas avant d'être portée?). Donc je ne le porte plus. J'y pose seulement mes doigts ou mes lèvres, presque tous les soirs, sauf quand je voyage au bout du monde, car, comme un animal il m'attend, bien caché dans sa cachette, à la maison, il ne voyage jamais. (Derrida 1998, 46, je souligne)

Le tallith de Derrida est "comme un animal», bien caché dans sa cachette, à la maison. On ne sais pas encore exactement s'il est un «animal» ou s'il est vivant, car le philosophe le précisera plus avant dans le texte, mais il est "comme un animal ", un animal domestique et pas du tout un animal nomade, destiné à l'errance ou à la «destinerrance» comme son maître Jackie ou comme un bélier en chair et os. Ce "comme un animal » déjà en captivité dans le "comme» de la similitude, ainsi que dans l'armoire, est donc domestique ou bien domestiqué, en tout cas il est effarouché, d'autant plus qu'il a à faire à la bénédiction, celle du père (Derrida 1998, 46), le maître/souverain par excellence, et à sa protection solennelle un peu étouffante, comme l'est la protection de sa cachette. En outre, ce tallith qui est «comme un animal » a à faire aussi à la mort. Mais à la mort de qui précisément? À sa propre mort en tant qu' "animal » ? À la mort du père? À la mort de l'autre, de celui qui est censé le porter sur soi comme un châle? À la mort de lui, Derrida, étant donné que ce tallith là est à lui seul? Nous verrons plus tard, dans le poème de Celan commenté dans Béliers, que la question du porter est essentielle: qui porte qui ? Qui porte l'autre? Ici, avant d'être porté comme un tallith, avant sa mort donc, ce «comme un animal» porte, quoi qu'il en soit, la Loi ou, selon les mots de Derrida, «il se porte en mémoire de la Loi », et Derrida $(1998,44)$ de citer $\mathrm{Nb} 15,39$ : «Ce sera votre frange, à vous, et quand vous la verrez, vous vous souviendrez de toutes les ordonnances de Iahvé, vous les exécuterez... ». Mais il y a plus. Dans ce commandement, celui de la frange, de la vue de la frange, Derrida voit une sorte de «prise de possession", mais 
dont la prise, voire la propriété est celle «de ce qui au fond n'appartient pas et n'est là que pour rappeler les commandements " (Derrida 1998, 44). "Comme si, explique-t-il, chacun découvrait son propre châle à sa propre vue, et à même son propre corps, mais seulement en vue d'entendre et de se rappeler la loi, de se rappeler à elle ou de la rappeler à soi " (Derrida 1998, 45). Or, qu'en est-il de «l'animal» dans cette «frange» qui, rien de moins, rappelle à soi-même et à la Loi ? Que reste-il de «l'animal», dans ce châle de la prière juive d'un homme? Derrida caresse son châle comme un animal (domestique, certes); il l'aperçoit seulement la nuit car la prière l'entoure, comme un animal de nuit; il s'enveloppe du châle comme une autre peau, comme la peau d'un animal; il s'inquiète si celui-ci vieillit en passant du blanc au jaune, comme la fourrure de l'animal... " comme un animal ", toujours. Mais il y a certainement déjà du vivant là-dedans, "quelque chose ", je dirais un peu maladroitement, de tellement vivant qui «fait naître à la parole divine qui l'aura précédé » (Derrida 1998, 45), «quelque chose» qui bouge, qui pousse, qui vit justement - qui, en fait, ne vit plus mais qui en quelque manière rappelle et relie à la vie - , qui est lié à la vie et même à la parole divine. Qui, dans sa vie même, relie de «liaison ou [d']alliance avec l'imprononçable» (Derrida 1998, 62). Quelle étrange bête est alors ce "comme un animal»?

Voyons, touchons ${ }^{8}$ plutôt. Car "avant tout, [son] tallith se touche», il se touche «comme les textes saints des tephillin» (Derrida 1998, 62, 63). $\mathrm{Au}$ tact, au toucher, mais surtout selon la prescription, c'est-à-dire selon la Loi qu'à son tour celui-ci rappelle, le tallith est et doit être tissé de «laine blanche de mouton» (Derrida 1998, 63). Plus précisément, écrit Derrida, "selon la Torah et les "ouvrages de décisionnaires", il semble que de brebis ou de bélier, la laine soit de rigueur» (Derrida 1998, 65 — je souligne). Et il ajoute pas après pas, à petits pas sans vouloir choquer ou surprendre, peut-être se surprendre lui-même par son idée: "laine": [...] un tissu animal, donc et, hier encore, à l'origine, un tissu vivant». Ce qui semble importer à ces "décisionnaires " dont nous ne savons rien mais auprès desquels Derrida fait son apprentissage quant à la matière du tallith et de la prière, en nous le transmettant à sa façon, comme un petit traité quant aux règles de la tallith-couture et de la vie, ce qui semble leur importer donc, à Derrida surtout, après la prière - celle-ci avant tout —, après la laine, après l'animalité, c'est précisément le «vivant »: «Ai-je assez insisté, écrit-il, sur ce qui m'importe ici, à savoir le vivant? Que commande en

8. Par rapport au toucher, voir Derrida et Hantaï (2000). 
premier lieu l'impératif catégorique de la laine et du cuir? Le poil et la peau: il faut que le tallith soit du vivant pris sur du vivant porté par du vivant. Mais, plus précisément, et plus tard, pris sur du mort qui fut un jour vivant, et ensevelissant un jour le mort qui fut naguère vivant. Vivant, c'est-à-dire qui aura eu quelque rapport à soi » (Derrida 1998, 66). Plus encore qu'à un vivant quelconque, le tallith touche à l'animal, il est animal et il n'est plus seulement un «comme un animal ", d'après ce que Derrida écrivait plus haut. Plus exactement, il est un animal bien précis, il est un bélier ou, en tout cas, appartenant à sa famille: «le tallith est d'abord animal, écrit-il. Comme les tefillins: une peau à même la peau. La peau ne venant pas de n'importe quel vivant animal, mais du mouton, de la brebis, du bélier, elle commémore de quelque façon une expérience qu'on appellerait sacrificielle si le mot de sacrifice n'était pas une mauvaise traduction pour korban (l'approche, le rapprochement) » (Derrida 1998, 67 — je souligne). Le tallith est alors, selon la séquence logique et analogique de ce texte: avant tout "comme un animal»; ensuite un «vivant» ou "du vivant »; puis, c'est-à-dire "d'abord », " animal »; enfin, il est un «bélier », bien que transfiguré à cause du korban.

Or, si cette séquence démontre déjà, comme je le disais, une certaine prudence face au risque de l'annonce de son hypothèse de la part de Derrida, à savoir, un peu absurdement, que le tallith est - et non pas représente - un bélier, elle montre aussi toute son attention, entièrement juive, au principe de la vie, au torat haïm ${ }^{9}$. C'est évident. Toutefois, il me semble qu'autant la séquence des mots que ce qui est dit dans ces passages surprenants expriment non pas tant les «songeries ", les bêtises ou bien les «impératifs» d'un philosophe (Derrida 1998, 66), que, plus audacieusement, le grand défi intellectuel le plus chimérique de Derrida et peut-être aussi son plus grand espoir, c'est-à-dire «la possibilité même de la promesse» (Derrida 1998, 66). Je m'explique. Ce fil blanc qui conduit du tallith au bélier ou plutôt, à l'envers, du bélier au tallith, et qui serait à comparer au fil blanc de la soie du récit d'enfance final sur le ver à soie, implique bien une transformation, une transfiguration: non tant la métamorphose du tallith en bélier, comme sur scène, sur cette première scène de mon propos; non tant la transformation manufacturière de la laine et

9. Derrida en rappelle l'importance dans un passage relatif à sa difficulté de comprendre la dénégation de «l'animal» chez Levinas, le plus juif parmi «ses» philosophes de référence, en écrivant ceci: «le principe de vie (torat haïm) reste un grand principe du judaïsme (bien qu'il n'ait jamais empêché le sacrifice animal dans le judaïsme énorme problème que je dois laisser de côté ici)»(Derrida et Mallet 2006, 155). 
de la peau d'un bélier en tissu pour le tallith, en autre peau (de laine et/ou de cuir) pour la prière, pour la bénédiction et la mort, sur laquelle insiste beaucoup Derrida; non tant, encore, la transfiguration du vivant en mort et du mort en vivant ou «du mort qui fut un jour vivant, et ensevelissant un jour le mort qui fut naguère vivant» (Derrida et Mallet 2006, 66), dans un jeu infini - celui de la vie - entre la vie et la mort, qu'une autre transfiguration encore. Tout cela, certes, est compris dans la transfiguration dont je parle. Mais il y a plus - et voici mon hypothèse qui redouble le risque de Derrida: il y est annoncé, en secret peut-être, le rêve, l'utopie, l'eschatos du «dernier des Juifs» (Derrida 1991, 133; 1996, 77-79; 2003b, 21), c'est-à-dire son effort ultime pour transfigurer le sacrifice en «rapprochement », en «approche». Cela est démontré d'abord par sa tentative plusieurs fois formulée - au moins chaque fois qu'il parle d'Abraham ou dudit "sacrifice d'Isaac» - de vouloir changer la traduction du mot korban, couramment traduit par "sacrifice», et le faire entendre au moins dans/par un mot plus proche tel «approche ", ou «rapprochement ». D'autant plus qu'il n'utilise jamais, à ma connaissance, le mot aquedah (ligature), qui au moins à propos d'Isaac serait plus correct, plus littéral ${ }^{10}$. Ce choix linguistique irait donc dans le sens de l'hypothèse d'une transformation conceptuelle basée sur une transformation linguistique. Mais ici, dans ce texte, vers la fin de «Un ver à soie», il n'est plus question uniquement de traduction, de substituer un mot par une autre, un concept par une autre, c'est-à-dire le sacrifice par le rapprochement; il s'agit plutôt d'une vraie transfiguration. Il l'écrit très discrètement, entre les lignes, très prudemment, quasi un murmure $^{11}$, quasi une prière du «quasi-juif» qu'il se veut: «quand la prière tend à remplacer, dans le "rapprochement", le sacrifice sanglant et la mise à mort du vivant, alors le châle de prière, le tallith et les tsitsith commémoreraient à la fois l'animal privilégié du sacrifice - la laine du mouton ou du bélier, le cuir etc. - et, pour aller jusqu'au terme eschatologique de l'histoire, d'un seul coup d'aile, le sacrifice du sacrifice, la fin du sacrifice dans le rapprochement, sa sublimation interminée et peut-être interminable, le rapprochement du

10. Deux fois seulement, à ma connaissance, Derrida utilise le mot "ligature", en se référant au livre biblique de Gn 22, mais n'utilisant jamais la translittération de l'hébreu aquedah (Derrida 2003a, 62-63). D'ailleurs, le mot korban qui en effet est formé sur la même racine que le mot "proche ", indique aussi les offrandes végétales, tandis que le terme réservé aux sacrifices d'animaux est zebah. Voir Chalier (1989, particulièrement le chapitre 4 "L'animalité et les sacrifices", 117-138).

11. Derrida $(1998,68)$ écrit en effet: "je continue à murmurer». 
rapprochement infini dans l'oraison de la prière" (Derrida 1998, 68). Derrida parle de «sublimation", et non pas de «transfiguration", mais le sens peut être le même, celui d'une transformation et d'une ascension en même temps, d'une transformation en élévation. Dans tous les cas, d'un changement et d'une montée. La sublimation contient en plus l'idée de purification, peut-être. Il est difficile de bien discerner les différences entre ces mots, mais celui de transfiguration serait plus lié à un langage théologique que celui de sublimation qui est lié à la chimie, d'où ma préférence ${ }^{12}$. Quoi qu'il en soit de cette distinction et de ce choix de l'interprète, que se passe-t-il alors dans la prière, grâce au tallith-bélier, grâce au «bélier de la prière» ? Qu'arrive-t-il ?

Rien de semblable à une révélation ou à la "gloire d'une vision lumineuse ", car tout ce passe dans la «nuit », dans la «nuit absolue », dit-il (Derrida 1998, 80). Une transfiguration dans la nuit. Quand le noir enveloppe le ciel, par la prière murmurée et presque chantée ${ }^{13}$ au toucher du tallith, "le sacrifice sanglant et la mise à mort du vivant» sont remplacés dans le «rapprochement» — à qui, sinon à Dieu? - grâce aux lèvres qui prient, grâce à la prière qui se lève, qui monte vers le haut. En même temps, mais «à contretemps» (Derrida 1998, 69), dans cette prière qui surgit au cœur de la nuit et au cœur le plus intime de Derrida, le bélier, en tant qu'animal élu pour et par le sacrifice, est commémoré; et, dans cette commémoration du bélier, faite aussi de "compassion sans limites et sans idolâtrie », faite par la «mémoire d'un événement à venir » (Derrida 1998, 80) Derrida célèbre, déjà, avant la fin de l'histoire, "la fin du sacrifice", l'eschatologique "sacrifice du sacrifice», dans son rapprochement à Dieu,

12. Le mot «transfiguration» évoque, en plus, celle du Christ selon Mt 17,1 qui se fait, comme une certaine transfiguration dans la prière de Derrida, en présence de Moïse et d'Élie - une présence-absence pour Derrida: de Moïse, à cause du rappel de la Loi, propre du tallith, comme nous avons vu, et d'Élie, car toujours présent dans l'acte de la circoncision, elle aussi évoquée ou rappelée par le tallith. Il y aurait certes beaucoup à dire à ce propos et au sujet des tentations «christiques " de Derrida.

13. Il vaut la peine encore de citer ce passage: "Je voudrais chanter la douceur très seule de mon tallith, la douceur plus douce que la douceur, toute singulière, sensible et insensible à la fois, calme, acquiesçante, étrangère à la sensiblerie, à l'effusion et au pathos, en un mot à toute "Passion". Compassion sans limite, cependant, compassion sans idolâtrie, proximité et distance infinie [...] Je l'aime et le bénis avec une étrange indifférence, mon tallith, dans une familiarité sans nom et sans âge. Comme si la foi et le savoir, une autre foi et un autre savoir, un savoir sans vérité et sans révélation se tissait ensemble dans la mémoire d'un événement à venir, le retard absolu du verdict, d'un verdict à rendre et qui se fait, se fit ou se fera venir sans la gloire d'une vision lumineuse. »(Derrida 1998, 79). 
dans «le rapprochement infini dans l'oraison de la prière». Car, comme l'écrit Catherine Chalier citant Os 14,3 et citée, elle, par Derrida: "la prière, cette "promesse de nos lèvres" destinée à "remplacer les taureaux [béliers]" doit être aussi agréable à Dieu que l'odeur des sacrifices" (Chalier 1982, 242; Derrida 1998, 68 $)^{14}$. Comme si la prière, celle des lèvres et du cœur, celle de Derrida également, pouvait, dans un «rapprochement» infini et interminable à Dieu, anticiper dans la promesse la promesse elle-même, celle faite à Abraham, celle d'un universalisme des singularités et des différences certes, mais aussi celle de la fin de l'effusion du sang, y compris le sang des toutes les créatures.

Mais nous sommes déjà trop loin, déjà dans la passion et dans l'avenir de la promesse, ainsi que dans la fin ultime, nous touchons déjà le bélier du sacrifice et celui de l'eschatologie.

\section{Seconde scène. «Le bélier du sacrifice»}

On sait très bien que Derrida réfléchit dans de nombreux textes à la scène biblique qu'on appelle trop rapidement le "sacrifice d'Isaac ", celle décrite en Gn 22 et qui, à son dire, devrait plutôt être nommée le «rapprochement d'Isaac» ou encore, différemment, le «sacrifice d'Abraham », bien qu'à mon avis elle devrait être nommée «le sacrifice du bélier "... Un livre entier est entièrement dédié à cette scène biblique: Donner la mort (1999), mais aussi des passages importants de Foi et savoir (1996, publié en 2000), de L'animal que donc je suis (1999, puis 2006), d' "Abraham, l'autre" (2003b), de «La mélancolie d'Abraham» (2004) (15 $^{15}$ et bien avant déjà, certaines pages de Glas (1974). Ne voulant pas traiter la question du sacrifice telle qu'elle est abordée dans tous ces textes ou en donner une nouvelle lecture, car cela me serait impossible d'une part et, de l'autre, certains commentateurs l'ont fait beaucoup mieux que je ne pourrais le faire, je continue, suivant mon propos animaliste-judaïsant, si ce couple peut tenir

14. Voir toute la partie III, Prier, parler, où Chalier évoque précisément la substitution des sacrifices par la prière qui n'as pas besoin d'un lieu et un temps bien précis, mais qui peut se faire, voire se dire dans tout lieu et à tout moment. Elle parle aussi de la prière comme de "rapprochement ", d' "approche" du transcendant, qui se donne comme proximité dans un ajournement incessant (Chalier 1982, 241-244). Derrida semble donc suivre de près cette lecture de Chalier.

15. Ainsi a été intitulée l'une des dernières entrevues de Derrida, réalisée au début de 2004 par Michal Ben-Naftali et publiée subséquemment (Derrida et Ben-Naftali 2012). A propos de la question du sacrifice, voir aussi le texte "Le sacrifice ", repris dans Derrida (2013). 
ensemble ${ }^{16}$, à suivre le bélier. Je reste alors cachée, car «tout cela se passe en secret» (Derrida 1999, 85): s'agissant de «l'épreuve du secret: pour l'un comme pour l'autre" (Derrida 1999, 163), je reste toujours derrière le bélier, laissant de côté, si cela est faisable, raisonnable et sans vouloir être blasphématoire, les deux autres protagonistes, Abraham et Isaac, en rappelant au passage, comme s'il y en avait la nécessité, que cette scène est la scène fondatrice du monothéisme juif.

Je voudrais en effet demander à Derrida, et avec lui : qu'arrive-il au bélier dans cette scène? "Nous devrions nous demander, inévitablement, écrit-il, ce qui arrive [...] à l'animal quand [...] un fils est après son père. Qu'arrive-t-il aux animaux de substitution ou non, à l'âne et au bélier sur le mont Moriah ?» (Derrida et Mallet 2006, 29) Mais quelle question bête! Tout le monde sait cela, même les bêtes... Pourtant, il faudrait leur demander, comme le suggère aussi le philosophe une seconde fois dans le même essai: "Demandez-le, dit-il, à l'âne et au bélier d'Abraham ou aux vivants qu'Abel sut offrir à Dieu: ils savent ce qui leur arrive quand des hommes disent "me voici" à Dieu, puis acceptent de se sacrifier, de sacrifier leur sacrifice ou de se pardonner» (Derrida et Mallet 2006, 52). Cependant, cette fois je m'adresse non pas aux bêtes, à l'âne et au bélier en particulier, mais à Derrida: en effet, même si les deux premiers ont un tel et commun savoir, est-ce la même "chose" - comme semble le suggérer un peu trop rapidement Derrida — «ce qui leur arrive ", "ce qui arrive » à l'un, à l'âne, redescendant à l'écurie manger son foin et suivant ses maîtres, le père et son fils épargné, et "ce qui arrive » au bélier, à celui qui après avoir été égorgé, brûle en holocauste à la place d'Isaac, et aujourd'hui encore sur le Moriah de tous les jours ${ }^{17}$ ? Mais j'ajoute une question pour Derrida qui va un peu dans le même sens que celle à peine formulée par lui : qu'en estil de ce bélier, le substitut d'Isaac, dans sa réflexion la plus argumentée sur ce sujet, c'est-à-dire dans Donner la mort? Car une seule fois il y est évoqué, et très rapidement (Derrida 1999, 171). Pourquoi ce sacrifice redoublé du bélier, dans ce livre en l'occurrence, portant justement sur le sacrifice, le secret, le sacré, le pardon, la décision et même la promesse? Pourquoi ce silence sur le sacrifice du bélier, mieux, sur le «bélier du sacrifice », ou plus généralement, sur le rôle de l'animal dans le sacrifice religieux, ici juif, dans

16. Je dis «si ", c'est-à-dire sous condition, car je songe à une phrase de Cixous (2003, 78 ) où elle raconte les propos de sa mère qui posait la question: «quels rapport les Juifs ont-ils avec les animaux? Les Juifs n'ont pas de rapport », paraît-il.....

17. «[C]hacun étant sacrifié à chacun sur cette terre de Moriah qui est notre habitat de tous les jours et de chaque seconde» (Derrida 1999, 99). 
un livre qui d'ailleurs a été écrit après d'autres ${ }^{18}$, ou presque en même temps, où la question du «devant l'animal » ou du vivant/animal était bien déjà annoncée, où la dénégation de «l'animot» était déjà dénoncée par Derrida lui-même?

Un exemple, seulement. Dans Donner la mort Derrida parle, on le sait, du tremblement, comme dans d'autres passages, en l'attribuant toujours à Abraham ou bien à soi-même. Or le tremblement ne devrait-il plutôt concerner le bélier dans le moment même de sa mise à mort ? Derrida écrit en effet ce passage un peu cryptique: «le tremblement [...] c'est, semblet-il, l'expérience du sacrifice. Non pas d'abord au sens hébraïque du terme, korban, qui veut dire plutôt l'approche et qu'on traduit abusivement par sacrifice, mais au sens où le sacrifice suppose la mise à mort de l'unique en ce qu'il a d'unique, d'irremplaçable et plus précieux. Il y va donc de la substitution impossible, de l'insubstituable, mais aussi de la substitution de l'animal à l'homme - et aussi, surtout, en cette impossibilité même, de ce qui lie le sacré au sacrifice et le sacrifice au secret " (Derrida 1999, 86). Ici, on le voit bien, plus ou moins bien, les idées se brouillent: Derrida ne se réfère pas directement au korban, au sacrifice de «l'animal», mais au sacrifice de l'homme dans le sens que d'abord et avant tout le sacrifice, dans cette scène de la Genèse, est celui d'Abraham qui se sacrifie, lui, qui renonce, en donnant son unique, son irremplaçable fils. Le sacrifice est donc, avant tout, celui d'Abraham: non pas celui d'Isaac, ni non plus celui du bélier. Le sacrifice de la bête est alors sacrifié par Derrida au nom du sacrifice d'Abraham; d'autant plus que, d'après lui, il y a et il y va d'une substitution «impossible» — celle d'Isaac —, concernant aussi, dans cette impossibilité même, la substitution de «l'animal». En d'autres termes, il me semble que paradoxalement Derrida nie en quelque manière ici à «l'animal », voire au bélier, son "droit» (son destin? son élection?) à la substitution sacrificielle — et jusque-là je le suivrais —, mais cela non pas au nom de la cause animale et pour elle, mais au nom de l'homme qui porte le nom d'Abraham et pour lui. Comme semblent le démontrer l'économie de l'ensemble du livre ainsi que ce bref passage, le centre de cette scène sacrificielle, où quand même un sacrifice a bien lieu, est Abraham et pas du tout, même pour une mince partie, même marginalement, le bélier. Bref, j'aurais aimé que Derrida s'en occupe ou le regarde un peu plus, étant donné que, comme maintes fois il le dit, «l'animal me regarde». Or, ce

18. Je pense à Derrida et Nancy (1992 — rédigé en 1989), Derrida (1998 — rédigé en 1995), Derrida et Mallet (2006 — rédigé en 1997). 
manque d'attention, voire cette ellipse du bélier, serait-il toujours redevable du même anthropocentrisme dont serait coupable non seulement le Kierkegaard qui est convoqué dans l'interprétation de cette scène et avec qui Derrida dialogue dans sa lecture, mais aussi, plus gravement, un anthropocentrisme dont serait affecté Derrida lui-même, le philosophe de l'animalité qui se définit comme «élu secret » (Derrida et Mallet 2006, 91), le philosophe élu des "animaux"? Si on répondait positivement à cette question, il serait trop facile de conclure que la déconstruction, censée dépister, surtout en philosophie, toute «structure sacrificielle» symbolique ou réelle propre à l'Occident, aurait échoué sur le point central de sa mise en question du propre de l'homme, en achoppant sur la scène de l'alliance abrahamique, bâtie sur la foi d'Abraham, mais aussi sur la scène du sacrifice par excellence, celui de «l'animal », ici le bélier "saisi au hallier ${ }^{19}$ " ou empêtré dans les buissons du mont Moriah. Même si je ne comprends pas au fond ce silence de Derrida quant à cette bête dans Donner la mort - et donc bien qu'un doute me reste à ce sujet - , je préfère plutôt penser que cet oubli, négation ou sacrifice du sacrifice de l'animal sacrificiel par excellence est dû à l'économie du sacrifice elle-même, dans le sens de la logique derridienne, à savoir qu'ici, dans ce livre, le philosophe met en acte, met en écriture, ce qui théoriquement et même eschatologiquement implique le sacrifice: il sacrifie le sacrifice. Bref, l'auteur de Donner la mort sacrifie, dans son écriture même, le sacrifice de «l'animal» et celui du bélier en l'occurrence. Mais pourquoi précisément?

Difficile de répondre, toujours difficile de trouver des réponses pour Derrida, c'est-à-dire à sa place, mais si on relit ce qu'il écrit dans une page de Foi et Savoir à propos de la religion, un pas de plus pourrait être fait dans une certaine direction. Derrida y écrit en effet:

la religion [...] est ellipse: l'ellipse du sacrifice. Pourrait-on imaginer une religion sans sacrifice et sans prière ? [...] là encore deux sources: la loi divisée, le double bind, le double foyer aussi bien, l'ellipse ou la duplicité originaire de la religion, c'est que la loi de l'indemne, le salut du sauf, le respect pudique de ce qui est sacro-saint (heilig, holy) exige et exclut à la fois le sacrifice, à savoir l'indemnisation de l'indemne, le prix de l'immunité. Donc l'autoimmunisation et le sacrifice du sacrifice. Celui-ci représente toujours le même mouvement, le prix à payer pour ne pas blesser ou léser l'autre absolu. Violence du sacrifice au nom de la non-violence. Le respect absolu

19. Selon la traduction de Gn 22,13 proposée par André Chouraqui (2003), que Derrida utilise toujours, avec celle d'Édouard Dhorme (1956). 
commande d'abord le sacrifice de soi, du plus précieux intérêt (Derrida et Wieviorka 2000, 80).

Si dans ce passage on retrouve certains thèmes repris successivement dans l'argumentation de Donner la mort, il me semble qu'on touche néanmoins à ce qui nous intéresse de plus près: pour le dire explicitement, le judaïsme en tant que religion et le rôle qui y est joué par «l'animal », par le bélier en particulier. D'abord, Derrida dit que la religion, toute religion, est ellipse: ellipse dans le sens de l'ellipse/omission du sacrifice, d'une part, mais aussi, d'autre part, dans le sens qu'elle aurait deux foyers ou deux sources — «la duplicité originaire de la religion», écrit-il — comme la figure géométrique de l'ellipse. Deux sources donc, qui seraient la prière et le sacrifice; mais aussi, à l'intérieur de ce dernier foyer (le sacrifice), deux foyers encore, soit l'exigence et l'exclusion à la fois du sacrifice, c'est-à-dire la nécessité de celui-ci et, en même temps, son ellipse, ou selon l'expression préférée par Derrida qui y recourt un peut partout et souvent, le «sacrifice $d u$ sacrifice». Or, le trois "grands monothéismes ", y compris l'Islam donc, "ont inscrit les alliances ou promesses donatrices dans cette épreuve de l'indemne qui est toujours une circoncision, qu'elle soit "extérieure ou intérieure", littérale ou comme cela fut dit avant saint Paul, dans le judaïsme même, "circoncision du cœur" "(Derrida et Wieviorka 2000, 75), explique Derrida toujours dans Foi et savoir. En laissant entendre tout ce qui est à entendre, à comprendre et à dire quant à la circoncision ${ }^{20}$, elle aussi sacrifice et même sacrifice par excellence, quelle soit celle du pénis ou du cœur, selon Dt10,12-11.32 (que Derrida évoque ici sans le citer ${ }^{21}$ ), sans faire donc ce grand détour autour de la circoncision en tant que sacrifice — il me semble important de souligner dans ce passage, où «l'épreuve de l'indemne» anticipe en tant qu'expression «l'épreuve du secret», c'està-dire dans le moment fondateur de l'alliance et de la promesse du Moriah, que cette épreuve-là, avant toute autre, nécessite et exclut le sacrifice, tout à la fois, d'où la dimension aporétique non seulement de toute religion, mais en particulier des religions abrahamiques ou ibrahimiques si on

20. Il nous suffit de renvoyer à Derrida 1974, 1980, 1986, 1991, où il parle explicitement de la circoncision, non seulement d'un point de vue biographique mais comme thème et philosophème décisifs pour comprendre toute son œuvre.

21. Mais dans Schibboleth, Derrida $(1986,106)$ cite à ce propos des références bibliques: la circoncision de la langue, selon Ex 6,12.30, celle des oreilles selon Jr 6,10 et finalement la circoncision du cœur d'après Lv 26, 41. 
inclut, comme on le doit, aussi la filiation ibrahimique d'Ismaël. Par conséquent, le judaïsme aussi, en tant que religion, et religion abrahamique en l'occurrence, nécessite du sacrifice et à la fois l'exclut: il nécessite du sacrifice intérieur ou de "la circoncision du cœur" d'Abraham, ainsi que du sacrifice extérieur de la chair du bélier et, en même temps, il nécessite son ellipse, sa négation, son exclusion, son sacrifice. En tout le cas, telle semble être la lecture, aporétique certes, de Derrida quant à la scène du mont Moriah, qui pourrait également justifier l'exclusion, voire l'ellipse, le sacrifice $d u$ sacrifice $d u$ bélier dans les pages de Donner la mort.

Or, de quelle manière tous ces discours sur le sacrifice dans les religions abrahamiques concernent-ils directement le bélier ${ }^{22}$, son sacrifice à lui, sa mise à mort, c'est-à-dire la mise à mort de l' "animal» en tant que tel par la main de l'homme? Y a-t-il une relation entre cette scène sacrificielle (et sa justification) et le sacrifice de "l'animal ", même après la fin des holocaustes religieux, suite à la destruction du second Temple (an 70 de l'ère chrétienne) ? Je ne peux que répondre positivement à cette question. Derrida est bien persuadé, en effet, que le sacrifice dit d'Isaac, voire le sacrifice d'Abraham (selon son interprétation), est lié aussi au sacrifice de "l'animal » en tant que tel, avec et par le sacrifice carnivore: "il y a un rapport entre tout ces sacrifices [...], — écrit-il dans l'un de ses derniers textes - le sacrifice d'Abraham, disons, et puis, le sacrifice carnivore, tel que j'en parle chaque fois que je parle de l'animal» (Derrida et Ben-Naftali 2012, 53). Et il précise: "D'après moi, il y a un lien entre la grande question canonique du sacrifice dit d'Isaac et le sacrifice carnivore. Je n'ai pas montré ce lien littérairement, mais il existe» (Derrida et Ben-Naftali 2012, 53). Bien qu'il ne l'ait jamais démontré « littérairement », il s'est au moins occupé de ce lien entre sacrifice religieux et sacrifice carnivore. C'est vrai. Au moins, il l'a évoqué chaque fois qu'il a parlé de «l'animal »; au moins, il l'a souligné quand il a pensé la religion; au moins, il l'a fait quand il a dénoncé l'incongruité désormais consolidée des religions abrahamiques et de la culture occidentale fondée sur celles-ci (sur le judaïsme et le christianisme), entre, d'une part, le commandement du «tu ne tueras point» et, d'autre part, la constante mise à mort du vivant ou de «l'animal», c'està-dire entre le respect absolu de la vie — celle de l'homme — et «la voca-

22. Derrida essaie de comprendre la question du sacrifice animal en se référant à l'épisode biblique de Caïn et Abel (Gn 4,1-15) où il s'agit de l'offrande agréable à Dieu : l'offrande animale d'Abel est préférée à celle végétale de Caïn qui, à cause de cela, tue son frère (Derrida et Mallet 2006, 67-69). Il aurait dû insister davantage sur cette piste. 
tion sacrificielle, elle aussi universelle " de la culture occidentale (Derrida et Wieviorka 2000,77ss). Au moins, encore, il l'a traité en relation à la question du sujet, à la «question du qui » (Derrida et Nancy 1992, 294), qui se construit, lui aussi, sur le sacrifice carnivore précisément et à partir d'un certain "carnophallogocentrisme ", selon ce que Derrida soutient très subtilement et très sensiblement dans l'entretien " "Il faut bien manger" ou le calcul du sujet» (1989), où il dénonce la «structure sacrificielle» de nos cultures qui s'auto-justifient du crime commis contre le vivant/ «animal» par l'idée d' "une mise à mort non criminelle », à savoir «avec ingestion, incorporation ou projection du cadavre. Opération réelle, mais aussi symbolique quand le cadavre est "animal" [...], opération symbolique quand le cadavre est "humain" " (Derrida et Nancy 1992, 293). Au moins, il l'a énoncé très explicitement, vers la fin de sa vie, dans l'interview intitulée postérieurement «La mélancolie d'Abraham »:

Il existe dans le sacrifice d'Abraham une certaine logique du sacrifice carnivore. Non seulement parce qu'il est prêt à tuer sa propre chair, ce qu'il aime le plus en lui-même, le fils de sa chair, et d'un faire le deuil, mais aussi parce que Dieu envoie l'ange qui signifie, à Abraham, le bélier comme possibilité de la substitution, c'est-à-dire la condition de possibilité du sacrifice. Il y a toujours substitution dans le sacrifice. Dans le sacrifice d'Abraham, comme dans le sacrifice carnivore, on substitue quelqu'un à soi-même et on engage ainsi une puissante structure dans laquelle le sacrifice carnivore puise son sens. (Derrida et Ben-Naftali 2012, 54-55, je souligne)

Nous aurions voulu entendre Derrida expliquer davantage ce lien ou cette logique qui relie le sacrifice d'Abraham au sacrifice carnivore, nous aurions voulu voir Derrida caresser un peu «à contre-poil» - comme dirait Walter Benjamin — ce bélier autour duquel cet enjeu se joue, où ce noue également l'extrême complexité de la structure sacrificielle fondant l'Occident (religieux et même philosophique) et toutes ses apories; nous aurions voulu en somme qu'il dégage littéralement ce lien qui serre et se serre comme l'enchevêtrement des cornes du béliers dans les buissons du Moriah. Mais peut-être là, Derrida nous aura laissé, à nous, une tâche essentielle, à suivre, celle de les dénouer, de les délivrer, de les libérer. Il nous aura laissé le devoir de penser "encore un autre Abraham» (Derrida 2003b, 42) et peut-être, avec celui-ci, la tâche de libérer le «bélier du sacrifice »: "non seulement Abram, puis Abraham, Abraham, deux fois ", y compris Ibrahim, comme écrit Derrida à la fin du texte "Abraham, l'autre ", mais aussi "encore un autre Abraham", c'est-à-dire encore un autre: 
l'Abraham qui écoute l'ange lui dire, comme s'il lui disait: Abraham! l'autre! Regarde l'autre, le tout autre, regarde-le, cet autre autre. "Encore un autre Abraham " : celui qui, appelé deux fois par l'ange de YHWH, par deux fois ne lève pas sa main sur l'autre, sur le bélier cette fois. Mais «qu'il y ait encore un autre Abraham, voilà donc la pensé juive la plus menacée mais la plus vertigineusement, la plus ultimement juive que je connaisse à ce jour. [...] Quand je dis "la plus juive", j'entends aussi "plus que juive”. D'autres diraient peut-être: "autrement juive", voire "autre que juive" » (Derrida 2003b, 42). À venir.

\section{Troisième scène. «Le bélier de l'eschatologie»}

Du ciel étoilé de la bénédiction faite à Abraham, où un double appel de l'ange de YHWH lui annonce une double bénédiction: "je te bénirai, je te bénirai, je multiplierai ta semence, comme les étoiles des ciels» (Gn 22,17), jusqu'au ciel noirâtre parsemé d'étoiles du poème «Grosse, Glühende Wölbung ${ }^{23}$ » de Paul Celan $(1967,93)$, commenté par Derrida (Derrida et Gadamer 2003) dans Béliers. Le dialogue ininterrompu: entre deux infinis, le poème: le ciel étoilé de la grande voûte incandescente est le scénario de la troisième scène où fait son apparition un autre bélier (bien qu'il soit toujours le même), où il s'agit également de sacrifice et de substitution, comme dans la scène du «bélier du sacrifice", mais aussi de prière et de bénédiction, comme dans celle du "bélier de la prière». En effet, dans ce petit livre, l'un de ses derniers, qui reprend la conférence que Derrida a donné en adieu à Hans-Georg Gadamer ${ }^{24}$ et où d'une certaine manière il s'explique après la mort du philosophe allemand, sur sa position différente quant à l'œuvre de Celan justement (Gadamer 1987), des pages remarquables sont consacrées au bélier, bien que le titre Béliers annonce en réalité une certaine pluralité. Déjà ce pluriel nous indique la voie, celle d'un singulier-pluriel, d'un toujours déjà singulier et pluriel en même temps, comme nous venons de voir, comme les deux autres béliers jusqu'ici ren-

23. Il vaut la peine de citer le poème pour mieux comprendre la lecture derridienne et celle de son interprète (traduction française de Jean-Pierre Lefebvre): "Grande voûte incandescente / avec / l'essaim d'astres noirs qui s'affouille / une voie de sortie, de départ: / au front caillou d'un bélier / je marque au feu cette image, entre / les cornes, dedans, / dans le chant des circonvolutions, enfle la / moelle des / mers de cœurs figées. / Contre/ quoi / ne fonce-t-il pas? / Le monde est parti, il faut que je te porte» (Celan et Lefebvre 2003, 113).

24. Conférence prononcée le 5 février 2003 à l’Université de Heidelberg. 
contrés nous l'ont indiqué dans leur manière de se montrer ou d'être à chaque fois uniques et aussi multiples, c'est-à-dire d'être singuliers et au singulier et en même temps d'évoquer l'autre, voire les deux autres, et ultimement d'être toujours pour l'autre. Mais ce singulier-pluriel est aussi à entendre dans le sens d'un devenir pluriel de la part de ce dernier bélier, qui se montre dans Béliers et que je nomme le «bélier de l'eschatologie», car il survient et donne sa sentence, selon le poème celanien, à la fin du monde, quand le monde n'est plus, dans un monde sans monde, sans monde et dans les temps ultimes. Il vient et se montre comme pour dire: "Die Welt ist fort, ich muss dich tragen (Le monde est parti, je dois te porter)».

Nous allons voir. Singulier-pluriel: bélier(s) précisément.

Le centre du poème de Celan, en tant que «figure allégorique, métonymique et théâtrale ", selon ce que Derrida rappelle dans une page de son dernier séminaire, est alors « une sorte de bête, un bélier (Widder) qui peut être à la fois ou successivement la constellation céleste ou le signe du zodiaque que l'on surnomme "bélier", le bélier, les béliers d'Abraham et d'Aaron dans des scènes dites sacrificielles bien connues ou le bélier de bois avec lequel on enfonce le mur» (Derrida, Lisse et al. 2008-2010, vol. II, 159 - je souligne), une sorte de bête qui a la chance de dire, d'être, par figure, tant de choses hétérogènes prises singulièrement et en même temps successivement, c'est-à-dire prises l'une après l'autre, à savoir un bélier après l'autre et ultimement se situant à la place de l'autre (et aussi pour le porter). Or, si cela est vrai, si ce bélier, ce Widder, porte en lui comme figure, d'autres béliers, s'il est à la fois unique et multiple, et non seulement dans le sens qu'il évoque ceux que nous avons suivis jusqu'ici - celui de la prière et du sacrifice - , il est pourtant indéniable que ce bélier-ci, qui rappelle encore des nouveaux béliers - par exemple, celui du zodiaque et celui utilisé comme machine de guerre - , est le plus juif parmi tous les autres. Et cela non tant parce qu'il apparaît sous la plume du plus juif parmi les poètes du $\mathrm{Xx}^{\mathrm{e}}$ siècle - et bien que pour Derrida, citant Celan qui cite en exergue Marina Tsvétaïeva, "tous les poètes sont des Juifs » (Derrida 1986, 91) —, que parce que ce bélier de Celan ou du Derrida de Béliers est le porteur par excellence et auquel le rapport au judaïsme est explicitement assigné. Il serait le plus juif et se montrerait comme tel. Cependant, il faut entendre qu'il est le plus juif, voire différemment juif, dans le sens que déjà Derrida utilisait presque vingt ans auparavant dans Schibboleth, où il rappelait que l'affirmation du judaïsme, que ce soit dans la formule «nous sommes Juifs» ou dans d'autres formulations comme 
celle-là ( «il est le plus juif»), voulait dire: "nous l'assumons, le prenons sur nous [...] et cela même si l'engagement ne se réduit pas à l'acte décisoire d'une volonté abstraite mais s'enracine dans la mémoire acceptée d'une destination non choisie» (Derrida 1986, 90, je souligne). C'était le sens du mot "Juif » du Celan de L'Entretien dans la montagne, repris par Derrida dans Schibboleth, dénonçant ou annonçant qu'il n'y aurait pas de propriété ni de spécificité juive: «le Juif, écrit Derrida, est aussi l'autre, moi et l'autre. Je suis Juif en disant: le Juif, c'est l'autre qui n'a pas d'essence, qui n'a rien en propre ou dont l'essence propre est de n'en point avoir ». Et il concluait: "le Juif est le schibboleth. Témoin de l'universel, mais au titre de la singularité absolue, datée, marquée, incisée, césurée - au titre et au nom de l'autre» (Derrida 1986, 91).

Dans cette direction, dans cette différence juive du schibboleth, qui envisage le Juif comme un "moi pour l'autre", toujours pour l'autre, il faut comprendre alors non seulement une certaine «assomption»/«affirmation» du judaïsme de la part de Derrida — de la part de celui qui a toujours voulu lire les poèmes de Celan "dans la nuit ${ }^{25}$ ", un peu comme il a toujours touché son tallith "dans la nuit", en pensant "avec lui vers lui » (Derrida 2003a, 26) —, mais il faut aussi entendre ce que je disais à propos du bélier, de ce bélier en particulier (Widder) comme du plus juif parmi ses semblables, dans ce sens différent, autre, ultime du mot «Juif». Le bélier, lui aussi, comme le Juif, et ce bélier en particulier en tant que le plus juif, serait alors "le témoin de l'universel au titre de la singularité absolue, au titre et au nom de l'autre ", c'est-à-dire que dans sa singularité d'individu / d'exemplaire il représenterait les autres de son genre, certes, mais surtout, ultimement - selon la lecture qui va suivre - il serait, audelà de tout exemplarisme, le témoin universel, dans cette différence, de la singularité juive au titre et au nom de l'autre. Car ultimement, à la fin ultime, quand le monde n'est ou ne serait plus, quand le monde serait déjà éloigné, selon le dernier vers de ce poème déjà cité, ce serait lui, le bélier, en tant qu' "animal », le bélier comme l'autre «animal», qui porterait sur soi et en soi l'autre, pour l'autre. Même si, peut-être, il le ferait au-delà de tout engagement, de tout choix, de toute volonté ou «acte décisoire », dans une passivité plus passive que toute passivité, selon une formulation lévi-

25. Outre Schibboleth. Pour Paul Celan (Derrida 1986), Derrida a commenté le poète allemand à quelques reprises (Derrida 2005; Derrida, Lisse et al. 2008-2010, vol. I, 289-313, 349-366). 
nassienne très connue, s'enracinant pourtant, comme le Juif, dans «une mémoire acceptée d'une destination non choisie».

Voyons. Écoutons. Plus haut, c'était le toucher, ici, il faut écouter.

En ouverture, la scène celanienne interprétée par Derrida est celle d'un ciel déjà habité "d'une vie animalière " (Derrida 2003a, 58), qui se meut d'une errance presque planétaire et compte parmi ses étoiles la constellation zodiacale du bélier, mais en passant seulement, comme en passant, et qui arrive à toucher de biais le "bélier belliqueux dont la ruée enfonce les portes ou rompt les murailles du château-fort (Mauerbrecher)» (Derrida 2003a, 58). Mais voici que, sous cette voûte d'une nuit planétaire animée, «le front silicifié d'un bélier» fait son apparition, dont le message serait à déchiffrer, ainsi que ce signe, ce sceau qui reste marqué sur son front, entre ses cornes. Et, encore, voici que ce museau presque pétrifié et pointé par ce signe quasi étoile, "l'étoile jaune ", se dessine et se calque davantage, page après page, ligne après ligne de ce livre:

Entre la vie la plus animale qui vient d'être plus d'une fois nommée et la mort ou le deuil qui hantent le dernier vers (Die Welt ist fort, ich muss dich tragen), le bélier, ses cornes et la brûlure rappellent et ravivent sans doute le moment d'une scène sacrificielle dans le paysage de l'Ancien Testament. Plus d'un holocauste. Substitution du bélier. Brûlure. Ligature d'Isaac (Genèse, XII). Après avoir dit une deuxième fois "Me voici », lorsque l'ange envoyé par Dieu suspend le couteau levé pour égorger Isaac, Abraham se retourne et voit un bélier pris par les cornes dans la broussaille. Il l'offre en holocauste à la place de son fil. Dieu promet alors de bénir et de multiplier sa semence comme les étoiles des cieux, peut-être aussi celle de la première strophe. Elles peuvent aussi devenir jusque dans le poème de terribles étoiles jaunes. (Derrida 2003a, 61-62, je souligne)

Dans ce passage donc, «le bélier du sacrifice», celui qui était épargné sur l'autel de la "logique" derridienne achoppant sur l'aporie de la structure sacrificielle de toute religion, et des religions abrahamiques en particulier, le bélier qui était négligé dans les pages de Donner la mort et dont l'absence m'inquiétait beaucoup, fait enfin son apparition avec, dans, sur, grâce à ce bélier «au front caillou » du poème de Celan. Comme si Derrida voulait ici réparer en quelque sorte son ellipse ou omission, en s'arrêtant et voyant cette fois, du même regard qu'Abraham, le bélier "pris par les cornes dans la broussaille» du mont Moriah. Toutefois, n'étant pas satisfait de ce témoignage donné au bélier d'Abraham et pour lui, et en rajoutant ou surenchèrant toujours, selon sa méthode d'écriture philosophique, Derrida sculpte mieux le profil, le visage, le museau de ce Widder. Le bélier 
du Moriah, en effet, tout en étant juif sans être uniquement juif, comme on l'a vu, ne suffit pas au philosophe pour entendre et comprendre entièrement le bélier celanien, plus juif que les autres, et il le rapproche en conséquence d'au autre bélier, celui d'Aaron, qui, dans une communauté de destin, fut immolé par le prêtre en expiation des fautes du peuple d'Israël, selon le récit biblique de Lv 16. Mais, dans ce dernier cas, dans cette "immense scène d'expiation pour les impuretés, les forfaits et les péchées d'Israël» (Derrida 2003a, 63), ce fût à un bélier d'être sacrifié, mais aussi à un bovillon, et encore, à deux boucs de chèvre, dont l'un était destiné à Dieu, l'autre, à un mystérieux Azazèl. Eux tous, furent immolés pour l'holocauste ou pour le «défauteur» (Chouraqui), c'est-à-dire pour l'expiation et la remise des péchés d'Israël. D'Israël seulement, et non pas des autres peuples. Ce bélier d'Aaron est donc encore plus circonscrit / spécifique en comparaison de celui d'Abraham et est limité à l'histoire des seuls Israélites et de leurs prêtres.

Mais il y a autre chose encore. La judéité de cet «animal» est à nouveau soulignée par Derrida, quand il s'arrête, admiratif, sur les spires de ses cornes:

les cornes du bélier deviennent cet instrument dont la musique prolonge un souffle et porte la voix. Dans ce qui ressemble à un chant ponctué comme une phrase, l'appel du schofar s'élève vers le ciel, il rappelle les holocaustes et résonne dans la mémoire de tous les Juifs du monde. Ce chant de joie déchirante est inséparable de la forme visible qui lui assure le passage [du poème]: des étranges spires, tours et détours, torsions et contorsion du corps de la corne. (Derrida 2003a, 63) ${ }^{26}$

De la même manière que le bélier du tallith, ce bélier du schofar ou qui est le schofar, dont les cornes rappellent et accueillent le souffle, la voix humaine et même celle de Dieu, ainsi que tous les holocaustes, scande la prière et la mémoire de tout Juif: non plus la prière quotidienne, faite au matin et au soir de chaque jour, mais la prière annuelle qui rythme le temps liturgique entre l'annonce du premier jour de l'an, Rosch haSchanah, et la fin de Yom Kippour. Comme le tallith, le schofar rappelle aussi - sans le rappeler —, le corps, le vivant, "l'animal», ainsi que son sacrifice et sa douleur dans «la torsion ou la contorsion du corps de la corne », et comme celui-là, il est, lui aussi, et sans être transfiguré, cet «animal» en particulier: le bélier. D'autant plus que le son qu'émet le schofar monte vers le

26. À propos du schofar, voir les pages de l'analyse de Mallet (1999a, 19-523), qui se base sur Reik (1974). 
ciel, de la même manière que la prière, des lèvres. Ainsi, tout Juif du monde associe le schofar

à la confession, à l'expiation, au pardon demandé, accordé ou refusé. Aux autres ou à soi-même. Entre deux dates fatidiques, entre le Jour de l'an et le jour du Grand Pardon, l'écriture de Dieu peut, d'une heure à l'heure, dans le livre de la vie, porter les uns et ne point porter les autres. Chaque juif [sic] se sent alors au bord de tout, au bord du tout, entre la vie et la mort, comme entre la renaissance et la fin, entre le monde et la fin du monde (Derrida 2003a, 63-64).

Le schofar comme le tallith, c'est-à-dire toujours le bélier, fait qu'on s'élève vers le ciel et il rapproche donc tout Juif de son Dieu, jusqu'à la fin du monde.

Or, après les cornes et le son, après le chant de leurs circonvolutions qui arrive jusqu'à Dieu, on croit entendre un mugissement, mieux un beuglement dans le vers suivant du poème celanien interprété magistralement par Derrida - «Wo- / gegen / rennt er nicht an? (Contre quoi ne fonce-t-il pas ?) ». Un beuglement qui n'évoque pas tant la souffrance de «l'animal» dans la violence du sacrifice, que la rage de la bête finalement, si l'on peut dire, ou la «ruée du bélier de bois", de la machine de guerre qui porte son nom, prête à forcer, presque en criant: «le bélier ne charge-t-il pas l'adversaire, un sacrificateur comme un mur, de tous les crimes?", demande Derrida (2003a, 65). Comme si, cette fois, dans ce vers, il y avait une rébellion de la part de cette bête et de tous les sacrifiés du monde:

on imagine la colère du bélier d'Abraham et d'Aaron, la révolte infinie du bélier de tous les holocaustes. Mais aussi, par figure, la rébellion violente de tous les boucs émissaires, de tous les substituts. Pourquoi moi ? [...] Le front de sa protestation jetterait le bélier contre le sacrifice même, contre les hommes et contre Dieu. Il voudrait mettre fin à leur monde commun. Le bélier chargerait contre tout et contre quiconque, dans toutes les directions, comme si la douleur l'aveuglait. (Derrida 2003a, 65-66)

Si le philosophe pouvait être ici suspecté de tentations antrophomorphiques, ce passage montre au contraire son audace et sa sensibilité interprétative, parce qu'il voit, il croit voir, il veut voir, le bélier agir - voire réagir -, même si la question du vers poétique est formulée négativement, et même si c'est au prix de l'hypothèse d'une révolte possible contre tous les sacrificateurs (dont nul n'est exclu), et donc aussi contre Dieu, aveuglé, lui aussi, par la douleur du monde. Peut-être. 
Et pourtant, ce bélier «au front silicifié » — qui devient un «front de protestation» endurci —, ce bélier qui pourrait attaquer, charger, foncer, qui voudrait dire adieu au monde, en demandant à l'homme et à Dieu "pourquoi moi ? ", ne charge pas. Il ne fait pas le pas de se révolter, d'attaquer, de mettre ses cornes contre l'homme et même contre Dieu. Dans le silence qui le sépare d'eux et dans celui de l'espace blanc qui conduit au dernier vers, il semble changer de position et accepter, à nouveau, à jamais, sa tâche, son destin, sa destination, son élection.

«Pourquoi moi ? », dirait alors le bélier, une deuxième fois, différemment, renversant les choses. Non plus «pourquoi moi ? ", en tant qu'objet de violence et de sacrifice, de souffrance et de meurtre, mais un "pourquoi moi ? » qui signifierait, dans une assignation hyperbolique, voire eschatologique, le devoir de se substituer à l'autre, le devoir de porter l'autre, le devoir d'être-pour-l'autre.

Cette question à double visage semble alors trouver une réponse dans le vers final: "Die Welt ist fort, ich muss dich tragen (Le monde est parti, je dois te porter)». C'est le vers que Derrida aime le plus ${ }^{27}$, comme «une signature peut-être eschatologique » dit-il (Derrida 2003a, 67), d'où mon choix de nommer ce bélier, «le bélier de l'eschatologie », comme si c'était sa signature à lui. Sa sentence à lui. Peut-être la dernière. Ce dernier vers serait alors la réponse à la question du bélier «pourquoi moi ? » posée par Derrida et à la question du poète dans le vers qui précède — «Wo- / gegen $/$ rennter nicht an? (Contre quoi ne fonce-t-il pas?) ", pourquoi ne foncet-il pas? En d'autres termes, le bélier répondrait à ces questions, se répondrait à soi-même, dans le vers final énoncé à la première personne, donc comme signataire de ce je dois te porter... Derrida l'entend aussi dans ce sens, comme possible signature du bélier, mais non pas seulement de lui ${ }^{28}$, car il écrit: "Quand le monde n'est plus [...], quand il n'est plus proche, quand il n'est plus ici (da), mais là (fort), quand il n'est même plus là (da) mais au loin parti (fort), peut-être infiniment inaccessible, alors je dois te porter, toi seul en moi et sur moi seul» (Derrida 2003a, 68, je souligne). Ou bien, encore, selon une autre direction: «je suis seul dans le monde dès

27. Derrida cite souvent ce dernier vers (voir au moins Derrida, Lisse et al. 2008-2010, vol. II, 31, 159-160; Derrida, Brault et al. 2003, 11; Derrida et Ben-Naftali 2012, 56).

28. Pour Derrida (2003a, 69), en fait, ce ne serait pas seulement le bélier le signataire de ce vers, mais aussi le bélier, avec les autres signataires, voire les protagonistes du poème: «le bélier, Abraham, Isaac, Aaron, la semence infinie de leur descendance, Dieu même, chacun s'adressant quand le monde est "fort" à la singularité absolue de l'autre». 
lors que je me dois à toi, que tu dépends de moi, que je te porte et dois assumer en tête à tête ou face à face, sans tiers, médiateur ou intercesseur, sans territoire terrestre ou mondial, la responsabilité à laquelle je dois répondre devant toi pour toi» (Derrida 2003a, 69). Dans la première direction interprétative, cette responsabilité infinie envers l'autre serait causée par un absentement, un éloignement du monde, voire par la fin du monde, tandis que dans la seconde direction, inversement, l'éloignement de celui-ci en serait la conséquence; dans les deux cas, cependant, s'annonce cette différence juive inscrite dans une singularité testimoniale consacrée à l'autre, que j'ai évoquée plus haut, ou bien se proclame l'asymétrie d'une responsabilité infinie envers l'autre, une responsabilité non choisie, mais assumée dans la passivité d'une élection, selon des échos thématiques d'Emmanuel Levinas, également présentes entre ces lignes. Une élection assumée, par «l'animal », une responsabilité infinie, de "l'animal». De la part de «l'animal», ici du bélier.

Paradoxale? Non, hyperbolique. Extrême. Ultime. Eschatologique. Comme si c'était à lui et non pas à l'homme, de porter l'autre, c'est-à-dire l'homme, lorsque l'éloignement du monde, presque un anéantissement de ce monde, devient proche et s'approche.

Alors "sur le bord eschatologique de cette limite extrême " (Derrida 2003a, 75), quand il n'y a plus de terre en dessous et quand il n'y que «l'altitude abyssale d'un ciel» au-dessus (Derrida 2003a, 68), il ne reste que lui, le bélier, «l'animal », pour dire l'éthique hyperbolique pensée par Derrida, une éthique au-delà de l'éthique, au-delà de toute éthique, au-delà de l'homme même, à la limite extrême, ultime, eschatologique précisément, celle que le bélier, lui, en tant qu' "animal » aurait tracé dans ce poème et qui se serait écrite par ces mots de Derrida: "Avant d'être, je porte, avant d'être moi, je porte l'autre. Je te porte et le dois, je te le dois. Je reste devant, en dette et devant à toi devant toi » (Derrida 2003a, 77, l'auteur souligne).

\section{Références}

Antonioli, M. et Jabre, E. (2014), dir., Bêt(is)es. Entre Derrida, Deleuze-Guattari et Sloterdijk, Chimères, 81.

Badmington, N. et Wolfe, C. (2007), Derridanimals, Oxford Literary Review, 29, Edimburg University Press.

Bensussan, G. (2012), "La bête est sans pourquoi », dans J. Cohen et R. Zagury-Orly, dir., Jacques Derrida. L'événement déconstruction, Paris, Gallimard (Les Temps moderne 669-670), p. 217-233. 
Berger, A.-E. et Segarra, M. (2011), Demenageries: Thinking (of) Animals after Derrida, Amsterdam/New York, Rodopi.

Caffo, L. et Ferraris, M. (2014), dir., Jackie D., Animot. L'altra filosofia, 1.

Calarco, M. (2008), Zoographies: The Question of the Animal from Heidegger to Derrida, New York, Columbia University Press.

Calarco, M., Filippi, M. et Trasatti, F. (2012), Zoografie: la questione dell'animale da Heidegger a Derrida, Milano, Mimesis.

Calle-Gruber, M. (1999), "Le fil de soie", dans M.-L. Mallet, dir., L'animal autobiographique: autour de Jacques Derrida [colloque, 11-21 juillet 1997, Centre culturel international de Cerisy-la-Salle], Paris, Galilée (La philosophie en effet), p. 61-85.

Celan, P. (1967), Atemwende, Frankfurt am Main, Suhrkamp [= référence suivante].

Celan, P. et Lefebvre, J.-P. (2003), Renverse du souffle. Édition bilingue, Paris, Seuil.

Chalier, C. (1982), Judaïsme et altérité, Lagrasse, Verdier (Les Dix Paroles). (1989), L'Alliance avec la nature, Paris, Cerf (La nuit surveillée).

Chouraqui, A. (2003), La Bible, Paris, Desclée De Brouwer.

Cimatti, F. (2013), Filosofia dell'animalità, Rome, Laterza.

Cixous, H. (2001), Portrait de Jacques Derrida en jeune saint juif, Paris, Galilée (Collection Lignes fictives).

(2003), "Ce corps étranjuif», dans J. Cohen et R. ZAGuryORLY, dir., Judéités, questions pour Jacques Derrida [actes du colloque international tenu du 3 au 5 décembre 2000 au Centre communautaire de Paris] [organisé par le Centre communautaire de Paris], Paris, Galilée (La philosophie en effet), p. 59-83.

DERrIDA, J. (1967), "Violence et métaphysique ", dans L'écriture et la différence, Paris, Seuil, p. 117-228. [= (1964), Revue de Métaphysique et de Morale, 69, p. 322-354 et 425-473]. (1974), Glas, Paris, Galilée.

(1980), La carte postale de Socrate à Freud et au-delà, Paris/ Montréal, Flammarion (Philosophie en effet). (1986), Schibboleth. Pour Paul Celan, Paris, Editions Galilée. (1991), «Circonfessions », dans G. Bennington et J. Derrida, Jacques Derrida, Paris, Seuil (Contemporains 11). 
(1996), «Un témoignage donné », dans E. Weber (dir.), Questions au judaïsme: entretiens avec Elisabeth Weber, [Paris], Desclée de Brouwer (Midrash), p. 73-104.

(1998), «Un ver à soie », dans H. Cixous et J. Derrida, Voiles, Paris, Galilée, p. 23-85.

(1999), Donner la mort, Paris, Galilée (La philosophie en effet). (2000), Le toucher, Jean-Luc Nancy, Paris, Galilée (Incises) (2003a), Béliers, le dialogue ininterrompu entre deux infinis, le poème, Paris, Galilée (La philosophie en effet).

(2003b), "Abraham, l'autre», dans J. Cohen et R. ZAGuryOrLy, dir., Judéités, questions pour Jacques Derrida [actes du colloque international tenu du 3 au 5 décembre 2000 au Centre communautaire de Paris], Paris, Galilée (La philosophie en effet), p. 11-42.

(2005), Poétique et politique du témoignage, Paris, L'Herne.

Derrida, J. et Ben-NAFtali, M. (2012), «La mélancolie d'Abraham (entretien) », dans J. Cohen et R. Zagury-Orly, dir., Jacques Derrida. L'événement déconstruction, Paris, Gallimard (Les Temps modernes 669-670), p. 30-66.

Derrida, J., Brault, P.-A. et al. (2003), Chaque fois unique, la fin du monde, Paris, Galilée.

Derrida, J., Lisse, M. et al. (2008-2010), dir., Séminaire La bête et le souverain, 2 vol., Paris, Galilée.

Derrida, J. et Mallet, M.-L. (2006), L'animal que donc je suis / édition établie par M.-L. Mallet, Paris, Galilée (La philosophie en effet).

Derrida, J., Michaud, G. et al. (2013), dir., Penser à ne pas voir - Écrits sur les arts du visible, 1979-2004, Paris, La Différence (Essais).

Derrida, J. et Nancy, J.-L. (1992), " "Il faut bien manger" ou le calcul du sujet ", dans J. Derrida, Points de suspension: entretiens choisis par E. Weber, Paris, Galilée, p. 269-301 [= (1989), Cahiers Confrontation, 20].

Derrida, J. et Wieviorka, M. (2000), Foi et savoir; suivi de Le siècle et le pardon (entretien avec Michel Wieviorka), Paris, Seuil.

Dhorme, É. (1956), La Bible: l'Ancien Testament, Paris, Gallimard (La Pléiade).

Fontenay, E. de (1998), Le silence des bêtes: la philosophie à l'épreuve de l'animalité, Paris, Fayard. 
(2008), Sans offenser le genre humain: réflexions sur la cause animale, Paris, Albin Michel (Bibliothèque Albin Michel idées).

Gadamer, H.-G., et Celan, P. (1987), Qui suis-je et qui es-tu?: commentaire de Cristaux de souffle de Paul Celan, Arles, Actes Sud.

Gontier, T. (2011), La question de l'animal: Les origines du débat moderne (Collection Hermann Philosophie).

Grondin, J. (2007), «Derrida et la question de l'animal », Cités, 30, p. 31-41.

Kofman, S. (1984), Lectures de Derrida, Paris, Galilée (Débats).

LAwlor, L. (2007), This is not Sufficient: An Essay on Animality and Human Nature in Derrida, New York, Columbia University Press.

Llored, P. (2012), Jacques Derrida politique et éthique de l'animalité, Mons, les Éd. Sils Maria (Cinq concepts).

Malabou, C. (2003), "La compulsion de révélation", dans J. Cohen et R. ZAGURY-OrLY, dir., Judéités, questions pour Jacques Derrida [actes du colloque international tenu du 3 au 5 décembre 2000 au Centre communautaire de Paris], Paris, Galilée (La philosophie en effet), p. 205-218.

Mallet, M.-L. (1999a), «Musique pour "l'animal que je suis" », dans M.-L. MALlET, dir., L'animal autobiographique: autour de Jacques Derrida [colloque, 11-21 juillet 1997, Centre culturel international de Cerisyla-Salle], Paris, Galilée (La philosophie en effet), p. 515-545.

Mallet, M.-L. (1999b), dir., L'animal autobiographique: autour de Jacques Derrida [colloque, 11-21 juillet 1997, Centre culturel international de Cerisy-la-Salle], Paris, Galilée (La philosophie en effet).

Michaud, G. (2009), «Sur une note serpentine», Lignes (Humanité Animalité), 28, p. 108-146. bel d'aujourd'hui).

Nancy, J.-L. (2014), Le dernier des Juifs, Paris, Galilée.

Ombrosi, O. (2010), «Ecce Animot: La Passion des animaux selon Jacques Derrida ", Archives de Philosophie, 73, p. 511-526.

(2012), "Non seulement un chien. Les bestiaires de Levinas et Derrida ", dans J. Cohen et R. Zagury-Orly, dir., Derrida. L'événement déconstruction, Les Temps Modernes, 669-670, p. 234-253.

ReIK, T. (1974), Le rituel. Psychanalyse des rites religieux, Paris, Denoël. 


\section{Résumé}

Dans cet article, je cherche à envisager un certain judaïsme de Derrida ou ce qui reste du judaïsme dans sa pensée, en suivant les traces qu'un animal — le bélier - laisse dans les écrits du philosophe. En effet, les deux sujets, celui de l'animalité et celui de la judéité se croisent et se côtoient précisément autour de cette figure animale, qui n'est pas pourtant une figure ou une métaphore uniquement. Je choisis donc de suivre le bélier pour suivre l'adresse de Derrida qui appelle les philosophes à répondre philosophiquement de l' "animal ", c'est-à-dire à s'interroger de la "question animale », d'une part; d'autre part et en même temps, je le suis pour voir les moments essentiels les plus représentatifs dans le rituel juif, où cet animal se montre, et pour discerner ce qu'il révèle justement en relation au judaïsme tel que le philosophe Derrida l'a vécu ou conçu. L'article se divise donc en trois grandes parties qui correspondent aux trois scènes où le bélier, les béliers plutôt, fait/font son/leur apparition: la première scène est celle du bélier de la prière, se montrant et se cachant dans le tallith; ensuite, la scène sacrificielle par excellence (Gen 22) où le bélier du sacrifice est sacrifié par Abraham; enfin, le bélier eschatologique, ainsi nommé parce qu'il est celui qui porte ou porterait l'autre, dans une dimension eschatologique à venir, selon une des dernières lectures derridiennes de Paul Celan, donnée dans le livre Béliers.

\section{Abstract}

In this article, I aim to contemplate Derrida's particular Judaism, or what remains of Judaism in his thinking, by following the tracks left by an animal - the ram - in the philosopher's writing. Indeed, the point where the two subjects of animalism and Judaism meet and intersect is precisely this animal image, which is nonetheless more than simply an image or a metaphor. I have therefore chosen to follow the ram firstly as a means of following the Derridean discourse which calls on philosophers to answer philosophically for the "animal", in other words to ponder the "question of the animal". At the same time, I will also follow this animal - the most characteristic in Jewish ritual - to find the crucial moments when it appears, and to identify what it reveals in relation to Judaism as experienced or conceived by Derrida. The article is thus divided into three major sections, which correspond to the three scenes where the ram - or rams - appear(s). The first scene is that of the ram in the context of prayer, hidden and revealed in the tallit; next is the quintessential sacrifice (Genesis 22) where the sacrificial ram is offered up by Abraham; and finally, the eschatological ram - so 
called because it carries or will carry the other into an eschatological dimension that is yet to come, according to one of Derrida's final interpretations of Paul Celan in the work entitled "Rams: Uninterrupted Dialogue Between Two Infinities, the Poem”. 